\title{
Strong magnon softening in tetragonal FeCo compounds
}

\author{
Ersoy Şaşığlu, ${ }^{*}$ Christoph Friedrich, and Stefan Blügel \\ Peter Grünberg Institut and Institute for Advanced Simulation, Forschungszentrum Jülich and JARA, 52425 Jülich, Germany
}

(Received 27 November 2012; published 29 January 2013)

\begin{abstract}
Magnons play an important role in fast precessional magnetization reversal processes serving as a heat bath for dissipation of the Zeeman energy and thus being responsible for the relaxation of magnetization. Employing $a b$ initio many-body perturbation theory we studied the magnon spectra of the tetragonal FeCo compounds considering three different experimental $c / a$ ratios, $c / a=1.13,1.18$, and 1.24 corresponding to FeCo grown on $\mathrm{Pd}$, Ir, and $\mathrm{Rh}$, respectively. We find that for all three cases the short-wavelength magnons are strongly damped and tetragonal distortion gives rise to a significant magnon softening. The magnon stiffness constant $D$ decreases almost by a factor of 2 from $\mathrm{FeCo} / \mathrm{Pd}$ to $\mathrm{FeCo} / \mathrm{Rh}$. The combination of soft magnons together with the giant magnetic anisotropy energy suggests $\mathrm{FeCo} / \mathrm{Rh}$ to be a promising material for perpendicular magnetic recording applications.
\end{abstract}

DOI: 10.1103/PhysRevB.87.020410

PACS number(s): 71.15.Qe, 71.45.Gm, 75.30.Ds, 71.20.Be

Since the introduction of the first commercial hard disk drive in 1956, the recording density in a hard disk, that is, the amount of information that can be stored per square inch, has increased by more than seven orders of magnitude to meet an ever-growing need. ${ }^{1}$ This has been achieved by a simple scaling of the dimensions of the bits recorded in storage medium. Due to the superparamagnetic effect, however, the recording density has an upper limit. For longitudinal magnetic recording it is around 200 Gbit per square inch, whereas it is predicted to be much larger for perpendicular recording, up to 1000 Gbit per square inch, though this limit is constantly changing with the discovery of new materials. ${ }^{2-4}$

The major problem in designing magnetic storage media is to retain the magnetization of the medium over a long period of time despite thermal fluctuations. If the ratio of the thermal energy $k_{\mathrm{B}} T$ to the magnetic energy per grain $K_{\mathrm{u}} V$, where $V$ is the grain volume and $K_{\mathrm{u}}$ is the uniaxial magnetocrystalline anisotropy energy (MAE), becomes sufficiently large, the thermal fluctuations can reverse the magnetization in a region of the medium, destroying the data stored there. ${ }^{3,5}$ In order to further increase the recording density in future recording media, high- $K_{\mathrm{u}}$ materials are needed. ${ }^{6}$ Additionally, a large saturation magnetization $M_{\mathrm{s}}$ is beneficial to reduce the write field, which has to be applied by the writing head. Materials that combine the desired large values of $K_{\mathrm{u}}$ and $M_{\mathrm{s}}$ are tetragonal near-equiatomic FeCo alloys. The large values of $K_{\mathrm{u}}$ and $M_{\mathrm{s}}$ in these alloys were first predicted by first-principles calculations ${ }^{7}$ and then confirmed by experiments. ${ }^{8-11}$ In particular, Yildiz et $a l .{ }^{11}$ achieved a strong perpendicular magnetic anisotropy (PMA) in tetragonal FeCo alloys epitaxially grown on $\operatorname{Pd}(c / a=1.13), \operatorname{Ir}(c / a=1.18)$, and $\operatorname{Rh}(c / a=1.24)$ substrates. The authors found that the PMA is very sensitive to the tetragonal distortion and increases with increasing $c / a$ ratio, which allows to tune the PMA by growing the alloys on different substrates.

Besides large $K_{\mathrm{u}}$ and $M_{\mathrm{s}}$ values, another very important issue in magnetic recording applications is the magnetic switching time, which imposes physical limits on data rates and areal recording densities. ${ }^{12}$ In current devices the switching speeds have reached a point where dynamical effects are becoming very important ${ }^{12-16}$ Magnons are created in fast (field driven) as well as ultrafast (laser induced) magnetization reversal processes. ${ }^{17-26}$ The former case is of particular interest for current device applications. It is found that above some threshold magnetic field the uniform precessional mode, i.e., the $\mathbf{k}=0$ magnons decay into nonuniform magnons $(\mathbf{k} \neq 0)$, i.e., the Zeeman energy stays in the magnetic subsystem and scatters between magnon modes. ${ }^{17-20}$ However, in ultrafast magnetization reversal the high-energy electrons generated by the laser field decay into the lower-energy magnon excitations. $^{22-24}$ In both cases spin-orbit coupling (SOC) is responsible for the transfer of the angular momentum to the lattice through different scattering mechanisms such as magnon-magnon, magnon-phonon, magnon-impurity scattering, and so on, where each process has a different relaxation time. ${ }^{27,28}$ The Landau-Lifshitz-Gilbert (LLG) equation with a phenomenological damping constant $\alpha$ is commonly employed to describe magnetization dynamics of small-angle precessional switching. ${ }^{29}$ However, recent studies have shown that in the case of large-angle (fast) switching, in which the magnons are created, the LLG equation should be extended in several aspects, ${ }^{30,31}$ in particular, a k-dependent damping constant $\alpha_{\mathbf{k}}$ has been proposed, ${ }^{32,33}$ which allows shortwavelength magnons to relax faster than those with $\mathbf{k} \rightarrow 0$. Thus, the magnetization relaxation processes, specifically, the damping of magnons, play an important role in designing ultrahigh-density magnetic recording media.

The aim of the present Rapid Communication is to study magnon dynamics in tetragonal FeCo compounds from first principles. Using a recently developed Green-function method $^{34}$ based on the many-body perturbation theory in the $G W$ approximation in combination with the multiplescattering $T$ matrix in a Wannier basis, ${ }^{35,36}$ we have calculated the dynamical spin susceptibility (DSS) of tetragonal FeCo compounds whose $c / a$ ratios were fixed to the experimentally determined values that relate to the three different substrates. As the unit cell contains two magnetic atoms, the calculated magnon dispersions exhibit two branches: an acoustic and an optical branch. The former persists throughout the Brillouin zone, indicating a localized nature of magnetism in FeCo compounds. The optical branch, on the other hand, is heavily damped due to the coupling to single-particle Stoner 
TABLE I. Lattice parameters $a$, spin magnetic moments $m^{\mathrm{s}}$ (in $\mu_{B}$ ), average screened on-site direct (diagonal) $\left(W^{\prime}=\frac{1}{5} \sum_{n}^{(3 d)} W_{n n ; n n}\right)$ and exchange $\left(J=\frac{1}{20} \sum_{m, n(m \neq n)}^{(3 d)} W_{m n ; n m}\right)$ Coulomb matrix elements (in $\mathrm{eV}$ ) between the $3 d$ orbitals, and magnon stiffness constants $D$ (in meV $\AA^{2}$ ) for tetragonal FeCo compounds grown on Pd, Ir, and Rh. Lattice parameters are taken from Ref. 11.

\begin{tabular}{|c|c|c|c|c|c|c|c|c|c|c|c|c|c|}
\hline & $a(\AA)$ & $c / a$ & $m_{[\mathrm{Fe}]}^{\mathrm{s}}$ & $m_{[\mathrm{Co}]}^{\mathrm{s}}$ & $m_{\text {[int] }}^{\mathrm{s}}$ & $m_{\text {[total] }}^{\mathrm{s}}$ & $W_{\mathrm{Fe}}$ & $W_{\mathrm{Co}}$ & $J_{\mathrm{Fe}}$ & $J_{\mathrm{Co}}$ & $D_{\|}$ & $D_{\perp}$ & $D_{\text {avg }}$ \\
\hline $\mathrm{FeCo} / \mathrm{Pd}$ & 2.75 & 1.13 & 2.81 & 1.79 & -0.12 & 4.48 & 1.68 & 1.75 & 0.52 & 0.54 & 470 & 650 & 560 \\
\hline $\mathrm{FeCo} / \mathrm{Ir}$ & 2.72 & 1.18 & 2.80 & 1.75 & -0.13 & 4.42 & 1.68 & 1.62 & 0.52 & 0.52 & 392 & 538 & 465 \\
\hline $\mathrm{FeCo} / \mathrm{Rh}$ & 2.69 & 1.24 & 2.80 & 1.73 & -0.13 & 4.40 & 1.68 & 1.49 & 0.52 & 0.51 & 206 & 372 & 289 \\
\hline
\end{tabular}

excitations. We find that the tetragonal distortion gives rise to significant magnon softening. The average magnon stiffness constant $D$ decreases almost by a factor of 2 from $\mathrm{FeCo} / \mathrm{Pd}$ to $\mathrm{FeCo} / \mathrm{Rh}$, which means that acoustic magnons can be excited much more easily in the latter material than in the former one. In field-driven fast magnetic switching processes, which take place on a time scale of ns to $100 \mathrm{ps,} \mathrm{the} \mathrm{excess}$ Zeeman energy will be transferred to the acoustic magnons and thus magnon stiffness constant $D$ and lifetime of $\mathbf{k} \neq 0$ magnons play a decisive role in determining the strength of the switching field and switching time. The latter is limited by the damping of magnons. Furthermore, damping also prevents the "backswitching" effect, which reduces the data rates in magnetic recording devices. ${ }^{37}$

To calculate the ground-state properties of the tetragonal FeCo compounds we use the full-potential linearized augmented plane-wave method as implemented in the FLEUR code. $^{38}$ The exchange-correlation potential is chosen in the generalized gradient approximation. ${ }^{39}$ The muffin-tin radii of the $\mathrm{Fe}$ and $\mathrm{Co}$ are chosen to be 2.29 a.u. A dense $16 \times 16 \times$ 16 k-point grid is used. The maximally localized Wannier functions are constructed with the WANNIER90 code. ${ }^{36,40}$ The DSS is calculated within a $T$-matrix approach ${ }^{35}$ as implemented in the SPEX code ${ }^{34}$ using $8000 \mathbf{k}$ points in the full Brillouin zone. We briefly review the method here. Within many-body perturbation theory the transverse DSS, $\chi^{-+}$, can be schematically written as $\chi^{-+}=\chi_{\mathrm{KS}}^{-+}+\chi_{\mathrm{KS}}^{-+} T^{-+} \chi_{\mathrm{KS}}^{-+}$, where the first term on the right-hand side represents the response of the noninteracting system, i.e., the Kohn-Sham DSS. The second term contains the $T$ matrix, which is given by $T^{-+}=\left[1-W \chi_{\mathrm{KS}}^{-+}\right]^{-1} W$, where $W$ is the screened Coulomb interaction. The $T$ matrix describes dynamical correlation in the form of repeated scattering events of particle-hole pairs with opposite spins and is responsible for the formation of collective magnon excitations. Details of the formalism, implementation, and applications to $3 d$ transition metals can be found in Ref. 35. The DSS provides complete information on the magnetic excitation spectrum including collective magnon modes as well as single-particle Stoner excitations together with their respective lifetimes. ${ }^{41-45}$ We note that magnon lifetimes and Stoner excitations are not accessible within the adiabatic approximation, a method mostly employed so far for the calculation of the magnon dispersion within density functional theory. ${ }^{46}$

Experimentally, FeCo alloys have been grown on the $\mathrm{Pd}$, Ir, and $\mathrm{Rh}$ substrates in the body-centered tetragonal structure, in which the in-plane lattice constant is determined by the substrate and the out-of-plane lattice constant changes so as to keep the volume constant. ${ }^{11}$ The experimental lattice parameters used in the calculations are presented in Table I. Yildiz et al. have shown that the structure remains stable for film thicknesses of up to 15 monolayers, which is large enough to consider it as a bulk in the context of theoretical modeling. ${ }^{47}$ Since no experimental information is available on the microscopic atomic order of FeCo alloys grown on the different substrates, we assume a tetragonally distorted CsCl-type (B2) structure derived from the known cubic bulk phase. We note that the mechanism behind the giant uniaxial MAE observed in tetragonal FeCo compounds has been discussed in detail in Ref. 7 and will not be dwelt on here. Indeed, our calculated values of uniaxial MAE (results not shown) are very similar to those reported by Burkert et al. ${ }^{7}$

We start with a discussion of the magnetic moments and the matrix elements of the screened Coulomb potential $W$. The latter are a crucial ingredient for the construction of the DSS. The calculated values for the three different $c / a$ ratios are presented in Table I. As seen, the spin magnetic moment of the Fe sublattice is substantially enhanced with respect to bulk bcc (or fcc) Fe, which has a magnetic moment of about $2.2 \mu_{B}$, while the Co sublattice shows moments that are more similar to the corresponding value of bulk Co, $1.62 \mu_{B}$. In contrast to bulk $\mathrm{Co}$, bcc $\mathrm{Fe}$ is a weak ferromagnet, and thus its magnetic moment is very sensitive to the local environment. The total spin magnetic moment of the unit cell is around $4.4 \mu_{B}$ and almost insensitive to the tetragonal distortion, which stems from the strong ferromagnetic nature of the FeCo compounds [see the inset in Fig. 1(a)]. Such a large magnetic moment is desirable for magnetic recording applications as it reduces the write field of the writing head. Not only magnetic moments but also the average screened on-site Coulomb matrix elements $W$ (direct) and $J$ (exchange) of FeCo compounds are insensitive
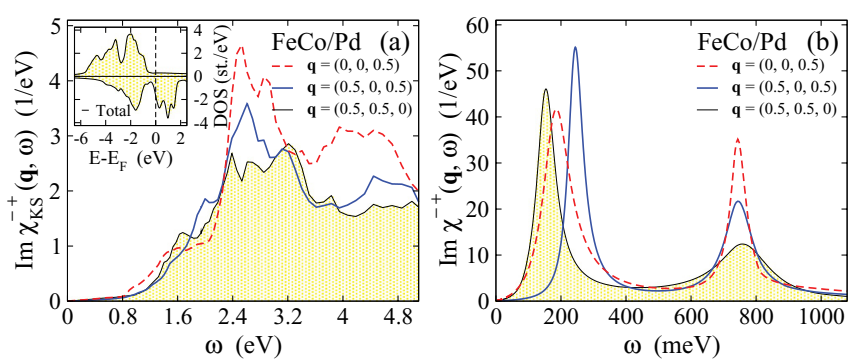

FIG. 1. (Color online) (a) Imaginary part of the unrenormalized Kohn-Sham spin susceptibility of the tetragonal FeCo compounds grown on $\mathrm{Pd}(c / a=1.13)$ for selected wave vectors. The inset shows the spin-resolved total density of states; (b) the same for the renormalized spin susceptibility. Note the different scales of the axes in the two figures. 
to the tetragonal distortion. The obtained values are slightly larger than the corresponding values in the bulk phase of bcc Fe and fcc Co. As discussed in detail in Ref. 35, this difference can be attributed to the larger exchange splitting of the $\mathrm{Fe}$ and $\mathrm{Co}$ atoms in $\mathrm{FeCo}$ compounds as the larger is the exchange splitting, the less screening takes place, leading to a stronger Coulomb interaction $W$. Indeed, with increasing $c / a$ ratio the magnetic moment (exchange splitting) of the Co atom decreases slightly, giving rise to a small reduction in the Coulomb matrix elements $W_{\mathrm{Co}}$, as shown in Table I.

Figure 1(a) presents the noninteracting Kohn-Sham transverse DSS of the tetragonal FeCo compounds grown on $\mathrm{Pd}$ $(c / a=1.13)$ for selected wave vectors at high symmetry points $\mathrm{M}[\mathbf{q}=(0.5,0.5,0)], \mathrm{Z}[\mathbf{q}=(0,0,0.5)]$, and $\mathrm{R}[\mathbf{q}=$ $(0.5,0,0.5)]$. As there is no dynamical correlation due to the absence of electron-electron interactions, only single-particle spin-flip Stoner excitations exist. As a consequence, the spectral function $\operatorname{Im} \chi_{\mathrm{KS}}^{-+}(\mathbf{q}, \omega)$ exhibits a broad peak originating from spin-flip transitions between occupied majority and unoccupied minority states. The peak maximum at around $2.5 \mathrm{eV}$ corresponds to the exchange splitting of the FeCo compounds visible in the density of states shown in the inset of Fig. 1(a). The situation is very similar for FeCo compounds grown on Ir and Rh. As will be discussed below, Stoner excitations are responsible for the damping of the magnons.

When dynamical correlation is included in the form of interacting spin-up and spin-down electrons via the screened Coulomb interaction $W$ (see Table I), additional magnon peaks appear in the spectral function of the interacting system $\operatorname{Im} \chi^{-+}(\mathbf{q}, \omega)$ at low energies, as illustrated in Fig. 1(b) for the case of $\mathrm{FeCo} / \mathrm{Pd}$. Since the unit cell contains two magnetic atoms, we obtain two modes: a low-energy acoustic mode and a high-energy optical mode. The former corresponds to the in-phase precession of the Fe and Co magnetic moments, while the latter is due to out-of-phase precession. The broadening of the peaks is caused by coupling to single-particle Stoner excitations. Plotting the renormalized susceptibility as a function of the wave vector yields the magnon dispersion as displayed in Fig. 2 along the high-symmetry lines in the Brillouin zone for all three systems. As seen in all cases, we obtain a well-defined low-energy acoustic branch throughout the Brillouin zone, while the high-energy optical branch lies above $0.75 \mathrm{eV}$ and is heavily damped due to coupling to Stoner excitations. This strong damping can be traced back to the density of states (DOS) of Stoner excitations in the respective energy region [see Fig. 1(a)]. We observe a drastic increase of the DOS above $0.8 \mathrm{eV}$. Below $0.8 \mathrm{eV}$ the DOS is small because the FeCo alloy is a strong ferromagnet, i.e., the spin-majority states are fully occupied. Thus, though damped, the optical branch remains identifiable in FeCo alloys, while in weak ferromagnets such as bcc Fe the magnons persist only up to $500 \mathrm{meV}$. Above this energy they disappear due to strong coupling to Stoner excitations. ${ }^{44,48}$ We note in passing that $s$ - $d$ mixing leads to damping of the magnons also in cases of strong ferromagnetism, but this effect is relatively weak compared to damping in weak ferromagnets. Damping of magnons does not mean that the angular momentum is transferred to the lattice. In the absence of SOC the angular momentum stays in the magnetic subsystem, i.e., it is transferred from magnons to the noncoherent single-particle spin-flip Stoner excitations.
The SOC is responsible for angular momentum transfer from the magnetic subsystem to the lattice subsystem. We also note that we have not included SOC in the calculation of the DSS. The SOC gives rise to an opening of a gap in the magnon dispersion at the $\Gamma$ point, whose value is determined by the MAE $K_{\mathrm{u}}$, which is less than $1 \mathrm{meV}$ for the systems considered here. ${ }^{7}$ Apart from that, we do not expect a qualitative change in the magnon spectra because the SOC only has a negligible effect on the electronic structure.

With increasing $c / a$ ratio the two branches are pushed in opposite directions, i.e., the excitation energy of the optical (acoustic) magnons increases (decreases). This behavior, on
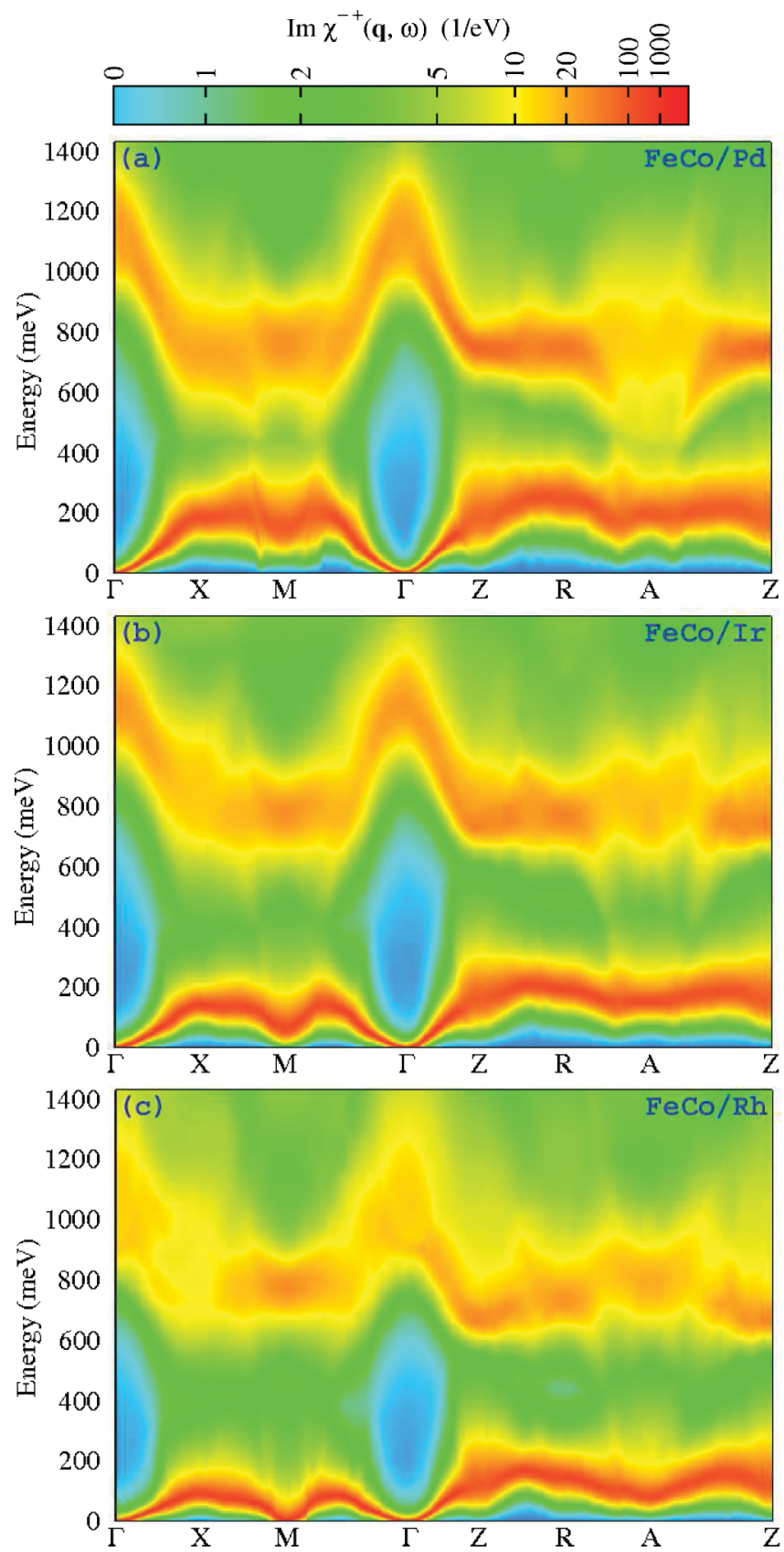

FIG. 2. (Color online) Magnon dispersion of the tetragonal ordered bulk FeCo alloy as grown on (a) $\mathrm{Pd}(c / a=1.13)$, (b) Ir $(c / a=1.18)$, and (c) $\mathrm{Rh}(c / a=1.24)$, along the high-symmetry lines in the Brillouin zone. 
the one hand, leads to weak damping of the acoustic magnons since the intensity of the Stoner DOS decreases at low energies, while the situation is just the opposite for the optical magnons. On the other hand, it gives rise to magnon softening, i.e., the magnon stiffness constant $D$ presented in Table $\mathrm{I}$ is considerably reduced with increasing $c / a$ ratio. As seen, from $\mathrm{FeCo} / \mathrm{Pd}$ to $\mathrm{FeCo} / \mathrm{Rh}$ the average $D$ decreases almost by a factor of 2 . The calculated average magnon stiffness constants $D$ for $\mathrm{FeCo} / \mathrm{Pd}$ and $\mathrm{FeCo} / \mathrm{Rh}$ are close to the experimental values of fcc $\mathrm{Co}\left(D=580 \mathrm{meV} \AA^{2}\right)$ and bcc Fe $\left(D=280 \mathrm{meV} \AA^{2}\right)$, respectively. ${ }^{49}$ Furthermore, the in-plane $\left(D_{\|}\right)$and out-of-plane $\left(D_{\perp}\right)$ magnon stiffness constants differ greatly, and this difference increases with increasing $c / a$ ratio. The small value and anisotropy of the exchange stiffness constant $D$ can be attributed to a strong direction dependence of the exchange interactions. ${ }^{50}$ Note that in itinerant ferromagnets there are several coexisting exchange interactions. A detailed discussion of them for the present systems is beyond the scope of the this work. In the following, we will discuss them only qualitatively. In $3 d$ ferromagnets and their alloys the total exchange coupling can be divided into two contributions: $J_{\mathrm{T}}=J_{\text {direct }}+J_{\text {indirect }}$, where the first term (direct coupling) is a short-range interaction due to the overlap of the $3 d$ wave functions and its strength depends on the distance between the magnetic atoms, while the long-range indirect part is due to the coupling of the localized $3 d$ moments to the itinerant $s p$ electrons. For $c / a \neq 1$ the $J_{\text {direct }}$ becomes anisotropic. With increasing $c / a$, i.e., from $\mathrm{FeCo} / \mathrm{Pd}$ to $\mathrm{FeCo} / \mathrm{Rh}$, the in-plane and out-of-plane components of $J_{\text {direct }}$ can differ greatly. The former (latter) is expected to increase (decrease) due to smaller (larger) interatomic distances. Consequently, the $c / a$ behavior of the direct exchange coupling can qualitatively account for the anisotropy of the magnetization and the reduction of the magnon energies (acoustic branch) along the $z$ direction in FeCo compounds. However, the strong in-plane magnon softening is more likely connected to $c / a$ behavior of the longrange indirect exchange interactions, which give a substantial contribution to the total exchange coupling $J_{\mathrm{T}}$ with a negative sign. A qualitative estimate of its contribution to $J_{\mathrm{T}}$ is not easy without a very detailed electronic structure analysis since this coupling shows Ruderman-Kittel-Kasuya-Yosida (RKKY)type oscillations, extends over very large distances, and is very sensitive to tetragonal distortion. ${ }^{50}$ Its strength and longrange behavior is determined by several parameters such as conduction electron spin polarization, Fermi surface topology, position of unoccupied states with respect to the Fermi level, and so on. For a detailed discussion on the indirect exchange coupling in $3 d$ transition metal alloys, the reader is referred to Ref. 51. Finally we would like to note that as the magnetism in itinerant ferromagnets depends on the electronic states far from the Fermi level, the disorder between Fe and Co sublattices is not expected to substantially influence the magnon spectra of FeCo compounds. ${ }^{52}$ However, this is not the case for MAE, which is very sensitive to the Fermi surface topology. ${ }^{53}$

In conclusion, we have calculated the magnon spectra of the tetragonal bulk FeCo compounds from first principles considering three different experimental $c / a$ ratios: FeCo grown on $\mathrm{Pd}$, Ir, and $\mathrm{Rh}$ with $c / a=1.13,1.18$, and 1.24 , respectively. We have found that for all three cases the short-wavelength magnons are strongly damped and tetragonal distortion gives rise to a significant magnon softening. The magnon stiffness constant $D$ decreases almost by a factor of 2 from $\mathrm{FeCo} / \mathrm{Pd}$ to $\mathrm{FeCo} / \mathrm{Rh}$, which reduces the switching field and yields efficient excitation of the $\mathbf{k} \neq 0$ magnons. Furthermore, the obtained strong damping of large-wave-vector magnons in FeCo compounds suggests a k-dependent damping constant $\alpha_{\mathbf{k}}$ in the LLG equation in describing magnetization dynamics of large-angle fast precessional switching. A combination of soft magnons with their substantial damping at large wave vectors as well as giant MAE suggests $\mathrm{FeCo} / \mathrm{Rh}$ to be a very promising material for ultrahigh-density perpendicular magnetic recording applications.

Fruitful discussions with $\mathrm{Ph}$. Mavropoulos, A. Schindlmayr, G. Bihlmayer, D. Bürgler, A. Kakay, and M. C. T. D. Müller are gratefully acknowledged.

\footnotetext{
*e.sasioglu@fz-juelich.de

${ }^{1}$ D. A. Thompson and J. S. Best, IBM J. Res. Dev. 44, 311 (2000).

${ }^{2}$ R. Fontana, N. Robertson, and S. Hetzler, IEEE Trans. Magn. 44, 3617 (2008).

${ }^{3}$ S. N. Piramanayagam, J. Appl. Phys. 102, 011301 (2007).

${ }^{4}$ N. Amos, J. Butler, B. Lee, M. H. Shachar, B. Hu, Y. Tian, J. Hong, D. Garcia, R. M. Ikkawi, R. C. Haddon, D. Litvinov, and S. Khizroev, PLoS One 7, e40134 (2012).

${ }^{5}$ D. Weller and A. Moser, IEEE Trans. Magn. 35, 4423 (1999).

${ }^{6}$ D. Weller, A. Moser, L. Folks, M. E. Best, W. Lee, M. Toney, M. Schwickert, J.-U. Thiele, and M. Doerner, IEEE Trans. Magn. 36, 10 (2000).

${ }^{7}$ T. Burkert, L. Nordström, O. Eriksson, and O. Heinonen, Phys. Rev. Lett. 93, 027203 (2004).

${ }^{8}$ G. Andersson, T. Burkert, P. Warnicke, M. Björck, B. Sanyal, C. Chacon, C. Zlotea, L. Nordström, P. Nordblad, and O. Eriksson, Phys. Rev. Lett. 96, 037205 (2006).

${ }^{9}$ F. Luo, X. L. Fu, A. Winkelmann, and M. Przybylski, Appl. Phys. Lett. 91, 262512 (2007).
}

${ }^{10}$ F. Yildiz, F. Luo, C. Tieg, R. M. Abrudan, X. L. Fu, A. Winkelmann, M. Przybylski, and J. Kirschner, Phys. Rev. Lett. 100, 037205 (2008).

${ }^{11}$ F. Yildiz, M. Przybylski, X.-D. Ma, and J. Kirschner, Phys. Rev. B 80, 064415 (2009).

${ }^{12}$ The Physics of Ultrahigh-Density Magnetic Recording, edited by M. Plumer, J. van Ek, and D. Weller (Springer, Berlin, 2001).

${ }^{13}$ P. B. Visscher, O. Traistaru, D. M. Apalkov, and X. Feng, J. Appl. Phys. 91, 7544 (2002).

${ }^{14}$ V. L. Safonov, J. Appl. Phys. 95, 7145 (2004).

${ }^{15}$ J. Fidler, T. Schrefl, W. Scholz, D. Suess, V. D. Tsiantos, R. Dittrich, and M. Kirschner, Physica B 343, 200 (2004).

${ }^{16}$ D. A. Garanin and H. Kachkachi, Phys. Rev. B 80, 014420 (2009).

${ }^{17}$ T. J. Silva, P. Kabos, and M. R. Pufall, Appl. Phys. Lett. 81, 2205 (2002).

${ }^{18}$ M. Feygenson, X. Teng, S. E. Inderhees, Y. Yiu, W. Du, W. Han, J. Wen, Z. Xu, A. A. Podlesnyak, J. L. Niedziela, M. Hagen, Y. Qiu, C. M. Brown, L. Zhang, and M. C. Aronson, Phys. Rev. B 83, 174414 (2011). 
${ }^{19}$ K. Lenz, H. Wende, W. Kuch, K. Baberschke, K. Nagy, and A. Jánossy, Phys. Rev. B 73, 144424 (2006).

${ }^{20}$ K. Baberschke, Phys. Status Solidi B 245, 174 (2008).

${ }^{21} \mathrm{Kh}$. Zakeri, J. Lindner, I. Barsukov, R. Meckenstock, M. Farle, U. von Hörsten, H. Wende, W. Keune, J. Rocker, S. S. Kalarickal, K. Lenz, W. Kuch, K. Baberschke, and Z. Frait, Phys. Rev. B 76, 104416 (2007).

${ }^{22}$ M. Djordjevic and M. Münzenberg, Phys. Rev. B 75, 012404 (2007).

${ }^{23}$ J. Walowski, G. Müller, M. Djordjevic, M. Münzenberg, M. Kläui, C. A. Vaz, and J. A. Bland, Phys. Rev. Lett. 101, 237401 (2008).

${ }^{24}$ A. Kirilyuk, A. V. Kimel, and T. Rasing, Rev. Mod. Phys. 82, 2731 (2010).

${ }^{25}$ B. Koopmans, J. J. M. Ruigrok, F. Dalla Longa, and W. J. M. de Jonge, Phys. Rev. Lett. 95, 267207 (2005).

${ }^{26}$ A. Manchon, Q. Li, L. Xu, and S. Zhang, Phys. Rev. B 85, 064408 (2012).

${ }^{27}$ R. Arias and D. L. Mills, Phys. Rev. B 60, 7395 (1999).

${ }^{28}$ M. C. Hickey and J. S. Moodera, Phys. Rev. Lett. 102, 137601 (2009).

${ }^{29}$ T. Gilbert, IEEE Trans. Magn. 40, 3443 (2004).

${ }^{30}$ A. Brataas, Y. Tserkovnyak, and G. E. W. Bauer, Phys. Rev. B 84, 054416 (2011).

${ }^{31}$ M. Fähnle and C. Illg, J. Phys.: Condens. Matter 23, 493201 (2011).

${ }^{32}$ E. M. Hankiewicz, G. Vignale, and Y. Tserkovnyak, Phys. Rev. B 78, 020404(R) (2008).

${ }^{33}$ S. Zhang and S. S.-L. Zhang, Phys. Rev. Lett. 102, 086601 (2009).

${ }^{34}$ C. Friedrich, S. Blügel, and A. Schindlmayr, Phys. Rev. B 81, 125102 (2010).

${ }^{35}$ E. Şaşıŏlu, A. Schindlmayr, C. Friedrich, F. Freimuth, and S. Blügel, Phys. Rev. B 81, 054434 (2010); E. Şaşığlu, C. Friedrich, and S. Blügel, ibid. 83, 121101(R) (2011).

${ }^{36}$ N. Marzari and D. Vanderbilt, Phys. Rev. B 56, 12847 (1997); F. Freimuth, Y. Mokrousov, D. Wortmann, S. Heinze, and S. Blügel, ibid. 78, 035120 (2008).
${ }^{37}$ S. Serrano-Guisan, K. Rott, G. Reiss, J. Langer, B. Ocker, and H. W. Schumacher, Phys. Rev. Lett. 101, 087201 (2008).

${ }^{38}$ http://www.flapw.de

${ }^{39}$ J. P. Perdew, K. Burke, and M. Ernzerhof, Phys. Rev. Lett. 77, 3865 (1996).

${ }^{40}$ A. A. Mostofi, J. R. Yates, Y.-S. Lee, I. Souza, D. Vanderbilt, and N. Marzari, Comput. Phys. Commun. 178, 685 (2008).

${ }^{41}$ S. Y. Savrasov, Phys. Rev. Lett. 81, 2570 (1998).

${ }^{42}$ K. Karlsson and F. Aryasetiawan, Phys. Rev. B 62, 3006 (2000).

${ }^{43}$ L. Ke, M. van Schilfgaarde, J. Pulikkotil, T. Kotani, and V. Antropov, Phys. Rev. B 83, 060404(R) (2011).

${ }^{44}$ P. Buczek, A. Ernst, and L. M. Sandratskii, Phys. Rev. B 84, 174418 (2011); B. Rousseau, A. Eiguren, and A. Bergara, ibid. 85, 054305 (2012).

${ }^{45}$ S. Lounis, A. T. Costa, R. B. Muniz, and D. L. Mills, Phys. Rev. B 83, 035109 (2011).

${ }^{46}$ M. Pajda, J. Kudrnovský, I. Turek, V. Drchal, and P. Bruno, Phys. Rev. B 64, 174402 (2001).

${ }^{47}$ A. Grechnev, I. Di Marco, M. I. Katsnelson, A. I. Lichtenstein, J. Wills, and O. Eriksson, Phys. Rev. B 76, 035107 (2007).

${ }^{48}$ A. Schindlmayr, C. Friedrich, E. Şaşığlu, and S. Blügel, Z. Phys. Chem. 224, 357 (2010).

${ }^{49}$ R. Pauthenet, J. Appl. Phys. 53, 8187 (1982).

${ }^{50}$ I. Galanakis and E. Şaşığlu, J. Phys. D: Appl. Phys. 44, 235001 (2011); V. D. Buchelnikov, V. V. Sokolovskiy, H. C. Herper, H. Ebert, M. E. Gruner, S. V. Taskaev, V. V. Khovaylo, A. Hucht, A. Dannenberg, M. Ogura, H. Akai, M. Acet, and P. Entel, Phys. Rev. B 81, 094411 (2010).

${ }^{51}$ E. Şaşığlu, L. M. Sandratskii, and P. Bruno, Phys. Rev. B 77, 064417 (2008); M. B. Stearns, Phys. Today 31(4), 34 (1978).

${ }^{52}$ R. M. Bozorth, Ferromagnetism (Wiley-IEEE, New York, 1993); M. Mašín, L. Bergqvist, J. Kudrnovský, M. Kotrla, and V. Drchal, arXiv:1212.4057.

${ }^{53}$ I. Turek, J. Kudrnovský, and K. Carva, Phys. Rev. B 86, 174430 (2012). 